\title{
Highlights of the season 2016-2017 by the Spanish Melanoma Group (GEM)
}

\author{
Ainara Soria ${ }^{1}$, Jose Luis Manzano ${ }^{2}$, Alfonso Berrocal ${ }^{3}$, Enrique Espinosa ${ }^{4}$, Ivan Marquez-Rodas ${ }^{5}$, Maria \\ Gonzalez-Cao $^{6}$, Salvador Martin-Algarra ${ }^{7}$; On behalf of the Spanish Melanoma Group (GEM) \\ ${ }^{1}$ Hospital Universitario Ramón y Cajal, Madrid, Spain; ${ }^{2}$ Hospital Germans Trias i Pujol, Badalona, Spain; ${ }^{3}$ Hospital General de Valencia, Valencia, \\ Spain; ${ }^{4}$ Hospital Universitario la Paz, Madrid, Spain; ${ }^{5}$ Instituto de Investigación Sanitaria Gregorio Marañón, Madrid, Spain; ${ }^{6}$ Translational Cancer \\ Research Unit, Instituto Oncológico Dr. Rosell, Hospital Universitari Dexeus, Quirónsalud Group, Barcelona, Spain; ${ }^{7}$ Clínica Universitaria de \\ Navarra, Pamplona, Spain \\ Correspondence to: María González-Cao, MD. Translational Cancer Research Unit, Instituto Oncológico Dr. Rosell, Dexeus University Hospital, \\ Quirónsalud Group, Barcelona 08028, Spain. Email: mgonzalezcao@oncorosell.com.
}

\begin{abstract}
In the last years there has been great progress in the treatment of advanced melanoma patients. This explosion of melanoma research has not declined, but rather has continued exponentially growing. In this review, the Spanish Group of Melanoma (GEM) compiles the highlights of melanoma treatment communicated or published between ASCO 2016 and AACR 2017. In this period, definitive data have been published about the possibility of achieving a long term survival with the use of single anti-PD-1 antibodies, as well as data on the description of clinical subgroups of patients that can also obtain a long term survival with the use of targeted drugs combining the oral BRAF and MEK inhibitors. The first immunotherapy combination including nivolumab, an anti-PD-1 antibody, plus ipilimumab, an anti-CTLA-4 antibody, has been approved, based on its demonstrated benefit in terms of overall survival versus ipilimumab alone.
\end{abstract}

Keywords: BRAF; CTLA-4; melanoma; PD-1

Submitted May 28, 2017. Accepted for publication Jun 08, 2017.

doi: $10.21037 /$ atm.2017.06.37

View this article at: http://dx.doi.org/10.21037/atm.2017.06.37

\section{Introduction}

In the past years, melanoma clinical research has led to the approval of new drugs by regulatory agencies. The results of recent clinical trials shed new light on the possibilities of a cure for some patients with advanced melanoma. In this review we try to summarize the most relevant findings between ASCO 2016 and AACR 2017 from a clinical point of view, which in all likelihood would soon impact the management decisions in the standard medical practice (Table 1).

\section{Latest advances in anti-PD-1/PD-L1 antibodies development}

Treatment with anti-PD-1 drugs is a well established therapy at the clinical setting and no major new data have been reported during the last year. In fact, most publications have consisted of clinical survival updates based on previously reported clinical trials and new safety data coming from increased experience at the clinical setting.

Pembrolizumab and nivolumab, both anti-PD-1 antibodies, are currently approved for the treatment of advanced melanoma patients. In the last year, data about overall survival benefit after a long follow-up were presented for both antibodies.

Final data of the phase III Keynote 006 trial, comparing at the first line setting the anti-PD-1 antibody, pembrolizumab to the anti-CTLA-4 antibody, ipilimumab, were presented at the last ASCO meeting. Pembrolizumab achieved at two years a survival rate of $55 \%$ and a progression free survival of $33 \%$, with a long durability of response $(70 \%$ of responders had a duration of response over 18 months) (1). At the same meeting, long term 
Table 1 Summary of the highlights of the season 2016-2017 by Spanish Melanoma Group (GEM)

\begin{tabular}{ll}
\hline Drugs & Relevant findings \\
\hline Pembrolizumab (1) & OS 2 years, 55\% \\
Pembrolizumab (2) & OS 3 years, $40 \%$ \\
Nivolumab (3) & OS 5 years, 35\% \\
Nivolumab + ipilimumab (4) & OS 2 years, 65\% vs. 54\% (HR 0.72, P=0.001)* \\
Atezolizumab + vemurafenib + cobimetinib (5) & OR 83\%; grade 3-4 toxicity 18\% \\
Atezolizumab + cobimetinib (6) & OR 40\%** \\
Epacadostat + pembrolizumab (7) & OR 58\%; grade 3-4 toxicity 18\% \\
Dabrafenib + trametinib (8) & OS 3 years, 45\% \\
Dabrafenib + trametinib by LDH level and less than three metastatic sites (9) & OS 2 years, 75\% vs. 7\% \\
Vemurafenib + cobimetinib (10) & OS 3 years, 37\% \\
Vemurafenib + cobimetinib by LDH level and liver metastasis (11) & OS 2 years, 89\% vs. 18\% \\
\hline
\end{tabular}

*, nivolumab plus ipilimumab versus ipilimumab; ${ }^{\star \star}$, BRAF wt. LDH, lactate dehydrogenase; OS, overall survival.

data from the phase I Keynote 001 study were presented. Keynote 001 is a trial that tested several different doses of pembrolizumab. Pembrolizumab at 3 years achieved a survival rate of $40 \%$, similar for all tested doses and independent of having received previous treatment with ipilimumab (2). Progression free survival at 3 years was $20 \%$ for the whole group, and $30 \%$ for the ipilimumab naïve patients. Also, in this update, responses were maintained during a long time, even after stopping treatment (of the 61 patients that interrupted treatment in complete response, only 2 had progressed) (2).

Similar data about long term survival were presented for the other approved anti-PD-1 inhibitor, nivolumab. At the 2016 AACR meeting, data from the phase I trial demonstrated an overall survival rate at 5 years of $35 \%$ (3). Updated results from the Checkmate 037 were also reported. This trial compared nivolumab versus chemotherapy after failure to ipilimumab. Updated data confirmed the response rate previously reported (12) and an absence of benefit in terms of overall survival versus chemotherapy, probably due to the use of subsequent treatments (13).

Quality of life data have been reported for both pembrolizumab and nivolumab. From the Keynote 002 trial comparing pembrolizumab versus chemotherapy for patients progressing to ipilimumab, global health status was similar in both groups at the beginning of the trial but it decreased in a greater magnitude throughout the treatment in the chemotherapy treated group (14). A similar trend was observed for individual functioning and symptoms scales. Quality of life for nivolumab has been assessed in the Checkmate 066 study comparing nivolumab and chemotherapy at first line setting. Patients on nivolumab maintained baseline quality of life as measured by the Euroquality of Life Five Dimensions for longer time and did not show increased symptom burden as assessed by the QLQ-C30 (15).

The second hit with single checkpoint inhibitors was the demonstration at the adjuvant setting of an overall survival benefit. Ipilimumab, an anti-CTLA-4 antibody, is the first drug that has demonstrated benefit in terms of overall survival in the adjuvant treatment of melanoma. The overall survival at five years was $65.4 \%$ in the ipilimumab group, as compared to $54.4 \%$ in the placebo group [hazard ratio (HR) for death, 0.72; 95\% CI, 0.58-0.88; $\mathrm{P}=0.001]$ (16). Grade $3-4$ toxicity occurred in more than $50 \%$ of patients treated with ipilimumab, so it generates concerns about the use of ipilimumab as an adjuvant treatment. Recently, data about quality of life from this phase III study has been published, demonstrating no differences between patients treated with ipilimumab versus control arm, despite this increase in toxicity (17).

Finally, this year several case reports communicated unusual forms of toxicity with these drugs. The toxicities have included acute myocarditis $(18,19)$, severe skin toxicity as Stevens Johnson syndrome (20), severe neurologic toxicities (21) and many other rare autoimmune toxicities as myasthenia gravis (22), sarcoidosis (23) or myositis (24) 
among others. Clinicians should be aware of these toxicities to identify them and to start early management.

On the other hand, increased experience has resulted in a better knowledge of the safety and efficacy of these drugs in selected special populations, such as patients with heart, kidney or liver impairment (25). Also anti-PD-1 antibodies demonstrated to be safe in patients with pre-existing autoimmune diseases or in patients that had suffered prior severe immune-related adverse events with ipilimumab (26). Patients who had been classically excluded from clinical trials can also benefit from anti-PD-1 therapies.

\section{Novel data about the combination of anti-PD-1 and anti-CTL-4 antibodies}

Primary resistance is observed in more than $50 \%$ of melanoma patients treated with single anti-PD-1 antibodies. The frequent co-expression of several targetable immune checkpoints in immune cells from cancer patients (27), support the rationale for testing at the clinical setting the combination of several checkpoints inhibitors. Since antibodies targeting different checkpoint inhibitors act at different levels of the immune response, synergy, or at least an additive effect, could be expected.

During the last few months, the first combination of checkpoint inhibitors for the treatment of cancer has been approved. The combination of ipilimumab, an antiCTLA-4 antibody, plus nivolumab, an anti-PD-1 antibody, demonstrated in the phase III clinical trial Checkmate 067 , an improvement in both progression free survival and overall response rate versus ipilimumab alone (28). With an extended follow-up, the combination was also superior to ipilimumab in terms of overall survival. The overall survival rate at 2 years was $63 \%, 59 \%$ and $45 \%$, for the combination arm, for the nivolumab, and for the ipilimumab arms, respectively (4).

The main problem with the combination of ipilimumab and nivolumab is the high rate of grade 3-4 toxicities, which exceeds $50 \%$ (4). In order to ameliorate it, some clinical trials are testing lower doses of ipilimumab $(1 \mathrm{mg} / \mathrm{kg})$. Results from the phase Ib trials of the combination of pembrolizumab and lower dose of ipilimumab, suggest a maintained activity with a lower toxicity (overall response rate was $57 \%$ and grade 3-4 toxicity was 42\%) (29). Results from the phase III clinical trial Checkmate 511, comparing the combination of nivolumab at $3 \mathrm{mg} / \mathrm{kg}$ with ipilimumab at $1 \mathrm{mg} / \mathrm{kg}$, versus the combination of nivolumab at $1 \mathrm{mg} / \mathrm{kg}$ with ipilimumab at $3 \mathrm{mg} / \mathrm{kg}$, will answer this question.

Also, a second way to ameliorate the toxicity of the combination could be the sequential administration of an anti-PD-1 and an anti-CTLA-4 drugs. Checkmate 064 was a trial designed to evaluate the optimal sequence between ipilimumab and nivolumab. Patients were randomly assigned to receive nivolumab $3 \mathrm{mg} / \mathrm{kg}$ every 2 weeks for six doses and then a planned switch to ipilimumab $3 \mathrm{mg} /$ $\mathrm{kg}$ every 3 weeks for four doses or the reverse sequence, both treatment arms continued after this induction with a maintenance phase of nivolumab (30). The primary objective was toxicity assessment and secondary endpoints were response and disease progression. No differences in toxicity were observed at week 25 between treatment arms. The nivolumab first arm demonstrated a response rate of $56 \%$, versus $31 \%$ for ipilimumab first (30). These data are preliminary, because a direct comparison of both schedules has not been performed, but it supports further research of this strategy based on the very similar data to the response rate $(61 \%)$ obtained with the concomitant combination of the two drugs (31).

Mucosal melanomas are infrequent tumors that are usually resistant to conventional therapies (32). A pooled analysis of 86 patients treated in clinical trials with nivolumab, ipilimumab, or with the combination, showed the largest evidence to date that immunotherapy in mucosal melanoma is an active treatment, especially for the combination of nivolumab and ipilimumab, that achieved a higher median progression free survival (5.9 versus 3 months) and overall response rate (37\% versus 23\%) than nivolumab alone (33).

\section{Combination checkpoint inhibitors with other drugs}

Data about the combination of immunotherapy with targeted drugs have been presented throughout the year, demonstrating interesting results. Preclinical data had demonstrated that the combination BRAF inhibitors plus MEK inhibitors increases the melanoma antigen expression, CD8+ T-cell infiltration, T-cell clonality, PDL1 expression and class I MHC up-regulation, while it decreased immunosuppressive cytokine release $(34,35)$. The problem came with the results from an initial phase I trial testing the concurrent combination of the BRAF inhibitor vemurafenib, with the anti-CTL4 antibody ipilimumab, that demonstrated a dose-limiting hepatotoxicity that led to an early termination of the trial (36). Also, in spite of 
the combination of the BRAF inhibitor, dabrafenib, with ipilimumab, showed no relevant toxicity, later, the triple combination of dabrafenib, ipilimumab and the MEK inhibitor, trametinib resulted also in dose-limiting toxicities, so the development of this combination was stopped (37).

The combination of targeted drugs with anti PD-L1 or anti-PD-1 antibodies seems more tolerable and active than the combination with anti-CTLA-4 antibodies. Last November at SMR, Sullivan et al. presented the results of a phase I trial testing the triple combination of vemurafenib, cobimetinib (a MEK inhibitor) and atezolizumab (an antiPD-L1 antibody), demonstrating a good tolerability and activity data. The overall response rate was $83 \%$ and grade 3-4 toxicity consisted mainly of increase of ALT (17\%), AST $(13 \%)$, creatinine phosphokinase (7\%) and diarrhea (7\%) (5). Actually, a phase III trial is on-going, comparing at the first-line setting the combination of atezolizumab, cobimetinib and vemurafenib versus the combination of vemurafenib and cobimetinib. Similar studies are planned comparing the combination of dabrafenib, trametinib and PDR001 (an anti-PD-1 antibody) versus the combination of dabrafenib and trametinib, as well as a phase II trial testing the combination of dabrafenib, trametinib and pembrolizumab (38).

Intriguingly, the combination of MEK inhibitors with anti-PD-1 or anti PD-L1 antibodies has recently demonstrated high activity in patients with BRAF non mutant melanoma. Infante et al. presented at the last SMR meeting the results of a phase Ib trial using the combination of atezolizumab plus cobimetinib. The Phase Ib study included 20 patients, 10 BRAF mutant and 10 BRAF wild type. Atezolizumab plus cobimetinib, showed an overall response rate of $50 \%$ in BRAF mutant and $40 \%$ in BRAF non mutant patients. Grade 3-4 toxicity was diarrhea in $14 \%$, acneiform dermatitis in $9 \%$, amylase increased in $5 \%$, anemia in 5\% and asthenia in 5\% (6). This synergical effect is based on the properties of cobimetinib promoting the tumor infiltration by $\mathrm{T}$ cells thanks to the inhibitory effect on the infiltrating immunosuppressive macrophages, M2 subtype (39).

Other interesting combinations included anti-PD-1 drugs with novel anti indoleamine 2,3-Dioxygenase (IDO) antibodies. IDO1 is an intracellular enzyme present in tumor cells, dendritic cells and B lymphocytes. It catalyzes the breakdown of trytophan to kynurenine, leading to immunosuppression by decreasing cytotoxic lymphocytes and expansion of the T-reg cells. There are several selective IDO1 inhibitors (indoximod, epacadostat, GDC0919) as well as an IDO peptide vaccine in clinical development. The IDO inhibitor indoximod was tested in 48 patients with advanced solid tumors as monotherapy (40). The most frequent adverse effects were grade 1-2 anemia, fatigue, anorexia, dyspnea, cough and nausea. Stable disease was achieved in $10 \%$ of patients with a duration of at least 6 months (40). At the 2016 ESMO meeting, Gangadhar et al. presented the results of the phase Ib trial (Keynote-037) exploring the combination of the IDO inhibitor epacadostat with the anti-PD-1 pembrolizumab. The phase Ib reported a favorable toxicity profile, with $18 \%$ of grade III/IV toxicities (consisting mainly of rash and increased lipase) and a response rate of $58 \%$ in treatment naïve patients. The real deal for this combo will be the confirmation of these results in the phase III clinical trial, recently completed, comparing the combination of pembrolizumab and epacadostat versus pembrolizumab and placebo (7).

About oncolytic virus for melanoma treatment, two works have been presented in AACR 2017. In the phase I KEYNOTE-200 (Storm study), an intravenously delivered oncolytic virus, Coxsackievirus A21 (CVA21), in combination with pembrolizumab, was tested in advanced cancer patients. CVA21 is an unmodified cold virus that over-expresses on cancer cells, with $88 \%$ of patients lacking anti-CVA21 serum antibody at baseline. CVA21 presents rapid cytoplasmic replication, which kills by oncolytic and immunotherapeutic mechanisms. In the first study, CVA21 in monotherapy was well tolerated with toxicity mainly consisting of fatigue and pyrexia grade I-II. One partial response was observed in 15 patients. The combination of CVA21 with pembrolizumab was also well tolerated and toxicity consisted of fatigue grade II in $20 \%$, and nausea grade II in $10 \%$ of patients (41). The second study tested the intratumoral administration of the oncolytic CVA2 1 and intravenous pembrolizumab in advanced melanoma. Overall, adverse events were low-grade, mainly consisting of constitutional symptoms related to CVA21 (fatigue, rash, anorexia, diarrhea). From the first 15 patients evaluable for response, the response rate was $60 \%$ ( $9 / 15$ patients) (42).

Talimogene laherparepvec (T-VEC) is a modified herpes virus genetically modified for producing granulocytemacrophage colony-stimulating factor (GM-CSF) in tumor cells. The GM-CSF recruits macrophages enhancing the tumor antigen presentation to $\mathrm{T}$ cells. This is the first oncolytic virus approved by the FDA and EMA for advanced melanoma (stage IIIC and M1a). The combination of T-VEC with check point inhibitors could be synergistic, based on preclinical data demonstrating 
an increased infiltration by CD8+ cells in lesions treated with intratumoral injection of T-VEC, that theoretically would enhance the anti tumor effect of the immune check points, such as anti-PD-1 (43) or anti-CTLA-4 antibodies (44). Last year's preliminary data showed a high activity of the combination of the anti-PD-1 drug, pembrolizumab, with T-VEC, with a response rate of $46 \%$ (45), and for the combination of T-VEC with ipilimumab, with a response rate of $50 \%$ (46).

\section{Targeted drugs: understanding their role}

BRAF and MEK inhibitor combinations have become a standard treatment for BRAF mutated melanomas due to the high response rate and the improvement in overall survival. An important benefit in terms of long term progression free and overall survival from different combinations have been reported throughout the year.

Long term data from both phase III clinical trials, COMBI-D (8) and COMBI-V (47), have demonstrated for the dabrafenib plus trametinib combination, a 3 -year progression free survival of $24 \%$ and a 3 -year overall survival of $45 \%$. Also for the combination of the BRAF inhibitor, vemurafenib, with the MEK inhibitor, cobimetinib, an improvement has been reported in terms of median overall survival versus vemurafenib monotherapy (22.3 versus 17.4 months, $\mathrm{P}=0.005$ ) in the coBRIM trial with a 3 -year overall survival rate of $37.4 \%(10,48)$.

The COLUMBUS trial, a phase 3 trial of encorafenib, an ATP-competitive BRAF kinase inhibitor, monotherapy versus its combination with binimetinib, an ATPuncompetitive inhibitor of MEK1/2, versus vemurafenib, also demonstrated a median progression free survival for the combination of 14.9 versus 7.3 months for patients treated with vemurafenib (HR 0.49, 95\% CI, 0.37-0.64; $\mathrm{P}<0.001$ ), and a response rate of $63 \%$ (49).

A pooled analysis of trials testing the combination of dabrafenib and trametinib (BRF113220, COMBI-D and COMBI-V) analyzed baseline factors from 617 patients (9). Patients with normal lactate dehydrogenase (LDH) levels and fewer than three metastatic organ sites had a 1- and a 2-year progression free survival of $68 \%$ and $46 \%$, respectively. Moreover, for this subgroup of patients, the 1- and 2-year overall survival rate was $90 \%$ and $75 \%$, respectively. On the other hand, patients with high $\mathrm{LDH}$ levels had 1- and 2-year progression free survival rate of $8 \%$ and $2 \%$, respectively and an overall survival rate of $40 \%$ at 1 year and $7 \%$ at 2 years (9).
A similar retrospective analysis was performed with the pooled data from the BRIM-2, BRIM-3, BRIM7 and coBRIM trials (11). For patients treated with the combination of vemurafenib and cobimetinib, LDH level was the most important prognostic factor: for those patients with normal levels and without liver metastasis, overall survival at 1 - and at 2 -year was $88.8 \%$ and $64.9 \%$. Those patients with high LDH levels, had overall survival at 1and 2 -year of $42.5 \%$ and $17.7 \%$, respectively (11).

Since some preclinical studies have shown that acquired resistance to BRAF inhibition can be reversible, a small phase II trial including 25 patients evaluated the activity of re-challenge with dabrafenib and trametinib in patients who had previously progressed on BRAF inhibitors (50). Patients should have previously progressed on BRAF inhibitors (with or without MEK inhibitors) and be off-treatment for at least 12 weeks. Retreatment with dabrafenib and trametinib obtained a partial response in 8 patients (32\%), and a stable disease in 10 patients (40\%) (50).

Other known driver mutations in melanoma included NRAS, GNAQ or c-kit, but there are no currently approved targeted therapies. Efficacy of MEK inhibition for NRAS mutant melanomas has been tested in the phase III NEMO trial, reported at the 2016 ASCO meeting (51). NRAS-mutant melanoma patients who were previously untreated or had progressed on or after immunotherapy were randomized (2:1) to receive either the MEK inhibitor binimetinib, or dacarbazine. Binimetinib significantly prolonged the median progression free survival $(2.8 \mathrm{vs}$. 1.5 months; HR $0.62,95 \%$ CI, $0.47-0.80 ; \mathrm{P}<0.001$ ) with no statistically significant differences in terms of OS (51).

\section{Conclusions}

In the last 5 years, new treatments have been developed for advanced melanoma patients. These new drugs can achieve benefit in terms of long-term survival. Basically, the treatments available fall into two broad groups: immunotherapy and targeted therapies. It is still a challenge to establish which treatment is the most appropriate for each case, but in the last year clinical and molecular predictors have been discovered that may help in the selection of treatments $(5,11,33,47)$. Likewise, the combination of checkpoint inhibitors, such as nivolumab and ipilimumab (52), or targeted drugs with immunotherapy (5), improves the activity, but it also produces an increase in terms of toxic effects (53). New treatment strategies based on early clinical data are 
especially promising, such as the combination of anti-PD-1 inhibitors with IDO inhibitors (7) or MEK inhibitors (41), in the case of non-mutated BRAF melanomas, as well as the triple combination of BRAF and MEK inhibitors with anti PD-L1 antibodies (54), in the case of mutated BRAF melanomas.

Important clinical advances are guaranteed in the upcoming years, and it is very possible that we can soon talk about cures for this disease that, just 5 years ago, barely had treatment opportunities.

\section{Acknowledgements}

None.

\section{Footnote}

Conflicts of interest: The authors have no conflicts of interest to declare.

\section{References}

1. Schachter J, Ribas A, Long GV, et al. Pembrolizumab versus ipilimumab for advanced melanoma: Final overall survival analysis of KEYNOTE-006. J Clin Oncol 2016;34 suppl:abstr 9504.

2. Robert C, Ribas A, Hamid O, et al. Three-year overall survival for patients with advanced melanoma treated with pembrolizumab in KEYNOTE-001. J Clin Oncol 2016;34 suppl:abstr 9503.

3. Hodi FS, Kluger H, Sznol M, et al. Abstract CT001: Durable, long-term survival in previously treated patients with advanced melanoma (MEL) who received nivolumab (NIVO) monotherapy in a phase I trial. AACR 107th Annual Meeting; 2016 April 16-20; New Orleans, LA, USA.

4. Larkin J, Chiarion-Sileni V, Gonzalez R, et al. Abstract \#CT075: Overall survival (OS) results from a phase III trial of nivolumab (NIVO) combined with ipilimumab (IPI) in treatment-naïve patients with advanced melanoma (CheckMate 067). AACR Annual Meeting 2017; 2017 Apr 2.

5. Hwu P, Hamid O, Gonzalez R, et al. Preliminary safety and clinical activity of atezolizumab combined with cobimetinib and vemurafenib in BRAF V600-mutant metastatic melanoma. Ann Oncol 2016;27:1109PD.

6. Infante J, Kim TM, Friedmann J, et al. Safety and clinical activity of atezolizumab combined with cobimetinib in metastatic melanoma. Presented at: SMR Annual Meeting; 2016 November 6-9; Boston, MA, USA.

7. Gangadhar TC, Hamid O, Smith DC, et al. Epacadostat plus pembrolizumab in patients with advanced melanoma and select solid tumors: Updated phase 1 results from ECHO-202/KEYNOTE-037. Ann Oncol 2016;27:1110PD.

8. Flaherty K, Davies MA, Grob JJ, et al. Genomic analysis and 3-y efficacy and safety update of COMBI-d: A phase 3 study of dabrafenib (D) + trametinib (T) vs $\mathrm{D}$ monotherapy in patients (pts) with unresectable or metastatic BRAF V600E/K-mutant cutaneous melanoma. J Clin Oncol 2016;34 suppl:abstr 9502.

9. Long GV, Grob JJ, Nathan P, et al. Factors predictive of response, disease progression, and overall survival after dabrafenib and trametinib combination treatment: a pooled analysis of individual patient data from randomised trials. Lancet Oncol 2016;17:1743-54.

10. Ascierto PA, McArthur GA, Dreno B, et al. Cobimetinib combined with vemurafenib in advanced BRAF(V600)mutant melanoma (coBRIM): updated efficacy results from a randomised, double-blind, phase 3 trial. Lancet Oncol 2016;17:1248-60.

11. Larkin JM, Ribas A, Flaherty K, et al. Identifying prognostic subgroups for outcomes in BRAFV600mutated metastatic melanoma patients (pts) treated with vemurafenib $(\mathrm{V}) \pm$ cobimetinib $(\mathrm{C})$ : A pooled analysis of BRIM-2, BRIM-3, BRIM-7 and coBRIM. J Clin Oncol 2016;34 suppl:abstr 9536.

12. Weber JS, D'Angelo SP, Minor D, et al. Nivolumab versus chemotherapy in patients with advanced melanoma who progressed after anti-CTLA-4 treatment (CheckMate 037): a randomised, controlled, open-label, phase 3 trial. Lancet Oncol 2015;16:375-84.

13. Larkin J, Minor D, D'Angelo S, et al. Overall Survival in Patients With Advanced Melanoma Who Received Nivolumab Versus Investigator's Choice Chemotherapy in CheckMate 037: A Randomized, Controlled, Open-Label Phase III Trial. J Clin Oncol 2017. [Epub ahead of print].

14. Schadendorf D, Dummer R, Hauschild A, et al. Healthrelated quality of life in the randomised KEYNOTE-002 study of pembrolizumab versus chemotherapy in patients with ipilimumab-refractory melanoma. Eur J Cancer 2016;67:46-54.

15. Long GV, Atkinson V, Ascierto PA, et al. Effect of nivolumab on health-related quality of life in patients with treatment-naive advanced melanoma: results from the phase III CheckMate 066 study. Ann Oncol 
2016;27:1940-6.

16. Eggermont AM, Chiarion-Sileni V, Grob JJ, et al. Prolonged Survival in Stage III Melanoma with Ipilimumab Adjuvant Therapy. N Engl J Med 2016;375:1845-55.

17. Coens C, Suciu S, Chiarion-Sileni V, et al. Health-related quality of life with adjuvant ipilimumab versus placebo after complete resection of high-risk stage III melanoma (EORTC 18071): secondary outcomes of a multinational, randomised, double-blind, phase 3 trial. Lancet Oncol 2017;18:393-403.

18. Läubli H, Balmelli C, Bossard M, et al. Acute heart failure due to autoimmune myocarditis under pembrolizumab treatment for metastatic melanoma. J Immunother Cancer 2015;3:11.

19. Johnson DB, Balko JM, Compton ML, et al. Fulminant Myocarditis with Combination Immune Checkpoint Blockade. N Engl J Med 2016;375:1749-55.

20. Saw S, Lee HY, Ng QS. Pembrolizumab-induced StevensJohnson syndrome in non-melanoma patients. Eur J Cancer 2017;81:237-9.

21. Tanaka R, Maruyama H, Tomidokoro Y, et al. Nivolumab-induced chronic inflammatory demyelinating polyradiculoneuropathy mimicking rapid-onset GuillainBarre syndrome: a case report. Jpn J Clin Oncol 2016;46:875-8.

22. Polat P, Donofrio PD. Myasthenia gravis induced by nivolumab therapy in a patient with non-small-cell lung cancer. Muscle Nerve 2016;54:507.

23. Cousin S, Toulmonde M, Kind M, et al. Pulmonary sarcoidosis induced by the anti-PD1 monoclonal antibody pembrolizumab. Ann Oncol 2016;27:1178-9.

24. Bilen MA, Subudhi SK, Gao J, et al. Acute rhabdomyolysis with severe polymyositis following ipilimumab-nivolumab treatment in a cancer patient with elevated anti-striated muscle antibody. J Immunother Cancer 2016;4:36.

25. Kanz BA, Pollack MH, Johnpulle R, et al. Safety and efficacy of anti-PD-1 in patients with baseline cardiac, renal, or hepatic dysfunction. J Immunother Cancer 2016;4:60.

26. Menzies AM, Johnson DB, Ramanujam S, et al. AntiPD-1 therapy in patients with advanced melanoma and preexisting autoimmune disorders or major toxicity with ipilimumab. Ann Oncol 2017;28:368-76.

27. Márquez-Rodas I, Cerezuela P, Soria A, et al. Immune checkpoint inhibitors: therapeutic advances in melanoma. Ann Transl Med 2015;3:267.

28. Larkin J, Hodi FS, Wolchok JD. Combined Nivolumab and Ipilimumab or Monotherapy in Untreated Melanoma. N Engl J Med 2015;373:1270-1.

29. Long GV, Atkinson V, Cebon JS, et al. Pembrolizumab (pembro) plus ipilimumab (ipi) for advanced melanoma: Results of the KEYNOTE-029 expansion cohort. J Clin Oncol 2016;34 suppl:abstr 9506.

30. Weber JS, Gibney G, Sullivan RJ, et al. Sequential administration of nivolumab and ipilimumab with a planned switch in patients with advanced melanoma (CheckMate 064): an open-label, randomised, phase 2 trial. Lancet Oncol 2016;17:943-55.

31. Postow MA, Chesney J, Pavlick AC, et al. Nivolumab and ipilimumab versus ipilimumab in untreated melanoma. $\mathrm{N}$ Engl J Med 2015;372:2006-17.

32. Shoushtari AN, Bluth MJ, Goldman DA, et al. Clinical features and response to systemic therapy in a historical cohort of advanced or unresectable mucosal melanoma. Melanoma Res 2017;27:57-64.

33. D'Angelo SP, Larkin J, Sosman JA, et al. Efficacy and Safety of Nivolumab Alone or in Combination With Ipilimumab in Patients With Mucosal Melanoma: A Pooled Analysis. J Clin Oncol 2017;35:226-35.

34. Liu $\mathrm{C}$, Peng $\mathrm{W}, \mathrm{Xu} \mathrm{C}$, et al. BRAF inhibition increases tumor infiltration by $\mathrm{T}$ cells and enhances the antitumor activity of adoptive immunotherapy in mice. Clin Cancer Res 2013;19:393-403.

35. Hu-Lieskovan S, Robert L, Homet Moreno B, et al. Combining targeted therapy with immunotherapy in BRAF-mutant melanoma: promise and challenges. J Clin Oncol 2014;32:2248-54.

36. Ribas A, Hodi FS, Callahan M, et al. Hepatotoxicity with combination of vemurafenib and ipilimumab. N Engl J Med 2013;368:1365-6.

37. Minor DR, Puzanov I, Callahan MK, et al. Severe gastrointestinal toxicity with administration of trametinib in combination with dabrafenib and ipilimumab. Pigment Cell Melanoma Res 2015;28:611-2.

38. Long GV, Hamid O, Hodi FS, et al. Phase 2 study of the safety and efficacy of pembrolizumab (pembro) in combination with dabrafenib (D) and trametinib (T) for advanced melanoma (KEYNOTE-022). 2016;34 suppl:abstr TPS9596.

39. Olson OC, Kim H, Quail DF, et al. Tumor-Associated Macrophages Suppress the Cytotoxic Activity of Antimitotic Agents. Cell Rep 2017;19:101-13.

40. Soliman HH, Minton SE, Han HS, et al. A phase I study of indoximod in patients with advanced malignancies. Oncotarget 2016;7:22928-38. 
41. Pandha HS, Harrington KJ, Ralph C, et al. Phase 1b KEYNOTE 200 (STORM study): A study of an intravenously delivered oncolytic virus, Coxsackievirus A21 in combination with pembrolizumab in advanced cancer patients. AACR Annual Meeting; 2017.

42. Silk AW, Kaufman HL, Gabrail N, et al. Phase Ib study of intratumoral oncolytic Coxsackievirus A21 (CVA21) and systemic pembrolizumab in subjects with advanced melanoma: Interim results of the CAPRA clinical trial. AACR Annual Meeting; 2017.

43. Daud AI, Loo K, Pauli ML, et al. Tumor immune profiling predicts response to anti-PD-1 therapy in human melanoma. J Clin Invest 2016;126:3447-52.

44. Spranger S, Spaapen RM, Zha Y, et al. Up-regulation of PD-L1, IDO, and T(regs) in the melanoma tumor microenvironment is driven by CD8(+) T cells. Sci Transl Med 2013;5:200ra116.

45. Long GV, Dummer R, Ribas A, et al. Efficacy analysis of MASTERKEY-265 phase 1b study of talimogene laherparepvec (T-VEC) and pembrolizumab (pembro) for unresectable stage IIIB-IV melanoma. J Clin Oncol 2016;34:abstr 9568.

46. Puzanov I, Milhem MM, Minor D, et al. Talimogene Laherparepvec in Combination With Ipilimumab in Previously Untreated, Unresectable Stage IIIB-IV Melanoma. J Clin Oncol 2016;34:2619-26.

47. Robert C, Karaszewska B, Schachter J, et al. Three-year estimate of overall survival in COMBI-v, a randomized phase 3 study evaluating first-line dabrafenib (D) + trametinib $(\mathrm{T})$ in patients (pts) with unresectable or metastatic BRAF V600E/K-mutant cutaneous melanoma. Ann Oncol 2016;27:LBA40.

48. McArthur GA, Dréno B, Atkinson V, et al. Efficacy of

Cite this article as: Soria A, Manzano JL, Berrocal A, Espinosa E, Marquez-Rodas I, Gonzalez-Cao M, Martin-Algarra S; On behalf of the Spanish Melanoma Group (GEM). Highlights of the season 2016-2017 by the Spanish Melanoma Group (GEM). Ann Transl Med 2017;5(19):391. doi: 10.21037/atm.2017.06.37
Long-Term Cobimetinib+ Vemurafenib in Advanced BRAF V600-Mutanted Melanoma: 3-year Follow-up of coBRIM (Phase 3) and 4-year Follow-up of BRIM-7 (Phase 1b). SMR Annual Meeting; 2016 November 6-9, Boston, MA, USA.

49. Dummer R, Ascierto PA, Gogas HJ, et al. Results of COLUMBUS Part 1: A Phase 3 Trial of Encorafenib (ENCO) Plus Binimetinib (BINI) Versus Vemurafenib (VEM) or ENCO in BRAF-Mutant Melanoma. SMR Annual Meeting; 2016 November 6-9; Boston, MA, USA.

50. Schreuer M, Jansen Y, Planken S, et al. Combination of dabrafenib plus trametinib for BRAF and MEK inhibitor pretreated patients with advanced BRAFV600-mutant melanoma: an open-label, single arm, dual-centre, phase 2 clinical trial. Lancet Oncol 2017;18:464-72.

51. Dummer R, Schadendorf D, Ascierto PA, et al. Binimetinib versus dacarbazine in patients with advanced NRASmutant melanoma (NEMO): a multicentre, open-label, randomised, phase 3 trial. Lancet Oncol 2017;18:435-45.

52. Hellmann MD, Rizvi NA, Goldman JW, et al. Nivolumab plus ipilimumab as first-line treatment for advanced nonsmall-cell lung cancer (CheckMate 012): results of an open-label, phase 1, multicohort study. Lancet Oncol 2017;18:31-41.

53. Gonzalez-Cao M, Boada A, Teixido C, et al. Fatal gastrointestinal toxicity with ipilimumab after BRAF/ MEK inhibitor combination in a melanoma patient achieving pathological complete response. Oncotarget 2016;7:56619-27.

54. Meyer C, Cagnon L, Costa-Nunes CM, et al. Frequencies of circulating MDSC correlate with clinical outcome of melanoma patients treated with ipilimumab. Cancer Immunol Immunother 2014;63:247-57. 